\title{
Proximity Effect and Josephson Coupling in the SO(5) Theory of High-Tc Superconductivity
}

\section{Citation}

Demler, E., A. J. Berlinsky, C. Kallin, G. B. Arnold, and M. R. Beasley. 1998. "Proximity Effect and Josephson Coupling in the SO(5) Theory of High-Tc Superconductivity." Physical Review Letters 80 (13) (March 30): 2917-2920. doi:10.1103/physrevlett.80.2917.

\section{Published Version}

10.1103/PhysRevLett.80.2917

\section{Permanent link}

http://nrs.harvard.edu/urn-3:HUL.InstRepos:28242606

\section{Terms of Use}

This article was downloaded from Harvard University's DASH repository, and is made available under the terms and conditions applicable to Other Posted Material, as set forth at http:// nrs.harvard.edu/urn-3:HUL.InstRepos:dash.current.terms-of-use\#LAA

\section{Share Your Story}

The Harvard community has made this article openly available.

Please share how this access benefits you. Submit a story.

\section{Accessibility}




\title{
Proximity Effect and Josephson Coupling in the SO(5) Theory of High- $T_{c}$ Superconductivity
}

\author{
E. Demler, ${ }^{1}$ A. J. Berlinsky, ${ }^{1}$ C. Kallin, ${ }^{1}$ G. B. Arnold, ${ }^{2}$ and M. R. Beasley ${ }^{3}$ \\ ${ }^{1}$ Department of Physics, Stanford University, Stanford, California 94305 \\ ${ }^{2}$ Department of Physics, University of Notre Dame, Notre Dame, Indiana 46556 \\ ${ }^{3}$ Department of Applied Physics, Stanford University, Stanford, California 94305
}

(Received 3 July 1997)

\begin{abstract}
We consider proximity effect coupling in superconducting-antiferromagnetic-superconducting sandwiches using the recently developed $\mathrm{SO}(5)$ effective theory of high temperature superconductivity. We find that, for narrow junctions, the $A$ region acts like a strong superconductor, and that there is a critical junction thickness which depends on the effective $\mathrm{SO}(5)$ coupling constants and on the phase difference across the junction, at which the $A$ region undergoes a Freedericksz-like transition to a state which is intermediate between superconductor and antiferromagnet. For thick junctions, the current-phase relation is sinusoidal, as in standard SNS and SIS junctions, but for thin junctions it shows a sharp break in slope at the Freedericksz point. [S0031-9007(98)05603-8]

PACS numbers: 74.50.+r, 75.70.-i
\end{abstract}

Zhang has recently developed a theory [1] which unifies $d$-wave superconductivity $(S)$ and antiferromagnetism $(A)$ on the basis of an underlying $\mathrm{SO}(5)$ symmetry. The $S$ and $A$ order parameters are combined into a five-dimensional superspin, and the high energy physics of these superspins is postulated to be rotationally symmetric. At low energies this $\mathrm{SO}(5)$ symmetry is broken by a chemical-potentialdependent anisotropy which favors the $A$ state for $\mu<\mu_{c}$ or the $S$ state for $\mu>\mu_{c}$. Here $\mu$ is the chemical potential, and $\mu_{c}$ is the critical value of the chemical potential at which the first order transition between the superconducting and antiferromagnetic states occurs. This implies that, at low temperature, there is a "soft direction" for perturbations of a stable $d$-wave superconductor toward antiferromagnetism. Similarly, the appropriate perturbation, applied to a stable $A$ material, will tend to drive it into the $S$ state. By analogy to the proximity effect in conventional superconductors, it is clear that the relevant perturbing field is provided by proximity of an $A$ material to an $S$ material. Moreover, in a sandwich superconductingantiferromagnetic-superconductivity $S-A-S$ configuration, this proximity effect would be expected to provide a mechanism for Josephson coupling of the two $S$ regions. We also note that one approach to practical high- $T_{c}$ Josephson junctions involves the use of barriers made from the cuprates near the $S / A$ transition.

In this paper we present analytic and numerical results for the properties of the $S-A-S$ Josephson junction system, shown on Fig. 1, in terms of $\mathrm{SO}(5)$ continuum theory in which the spatial variation of the order parameter is one dimensional. We obtain analytical results for the critical current as a function of thickness and numerical results for the current-phase relation for different thicknesses.

We find that when the $S$ layers are strongly superconducting, thin $A$ layers are driven completely superconducting by the field of the adjacent $S$ layers, and the $\mathrm{SO}(5)$ order parameter lies completely in the superconducting plane.
Beyond a critical barrier thickness, we find that the order parameter in the junction starts to tip back toward the antiferromagnetic plane, in a fashion precisely analogous to the Freedericksz transition in liquid crystals [2]. Twisting the superconducting phase, which causes a current to flow through the junction, is analogous to twisting the nematic director at the walls. A sufficiently large twist will drive the system through the Freedericksz transition resulting in a distinctive, nonsinusoidal current-phase relation for an $S-A-S$ junction.

Our results clearly demonstrate that, within $\mathrm{SO}(5)$ theory, the details of Josephson coupling through an $A$ barrier are qualitatively different from those of proximity effect junctions with conventional barriers. Hence study of $S-A-S$ junctions provides a critical test of $\mathrm{SO}(5)$ theory. By the same token our calculations provide a new basis for the interpretation of real high- $T_{c}$ Josephson junctions, currently being fabricated and studied [3-5].

In the spirit of $\mathrm{SO}(5)$ we describe the system by a three-component order parameter $\mathbf{n}=\left\{n_{x}, n_{y}, n_{z}\right\}$, where the first two components are the real and imaginary parts of the superconducting order parameter and the third component represents the antiferromagnetic Neel vector (see Fig. 2). For simplicity we treat the Neel vector as a single component. However, this component may be viewed as the spatially varying amplitude of a $3 \mathrm{D}$ vector whose direction is uniform in the sample.

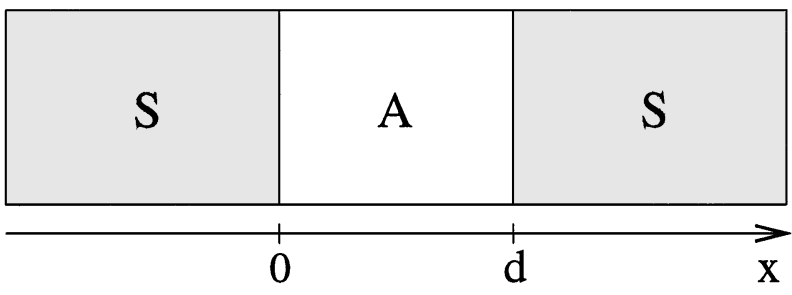

FIG. 1. Geometry of the suggested junction. 


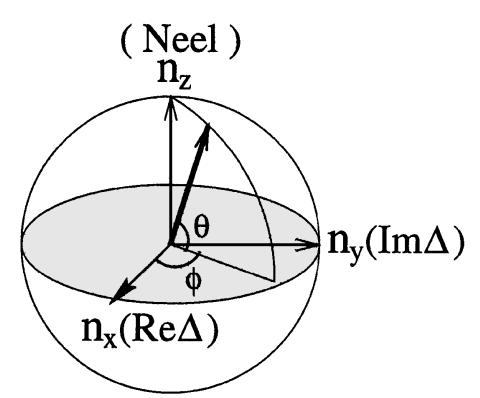

FIG. 2. $\mathrm{SO}(5)$ order parameter.

According to [1] the system is described by a functional

$$
\mathcal{L}(\mathbf{n})=\int d x\left\{\frac{\rho}{2}\left(\partial_{\mu} n_{a}\right)^{2}-g n_{z}^{2}\right\}
$$

with the constraint $n^{2}=1$. As in [6] we assume that the gradient term is $\mathrm{SO}(5)$ symmetric. The anisotropy term $g$ is positive in the $A$ region (so that it would be antiferromagnetic in the absence of proximity effects) and negative in the superconductor [7].

The superspin constraint is most naturally implemented in polar coordinates $n_{x}=\cos \theta \cos \phi, n_{y}=\cos \theta \sin \phi$, and $n_{z}=\sin \theta$.

$$
\begin{gathered}
\mathcal{L}(\theta, \phi)=\int d x\left\{\frac{\rho}{2}\left[\left(\partial_{\mu} \theta\right)^{2}+\cos ^{2} \theta\left(\partial_{\mu} \phi\right)^{2}\right]\right. \\
\left.-g \sin ^{2} \theta\right\} .
\end{gathered}
$$

In all of our calculations we will assume rigid superconducting boundary conditions $\left.n_{z}\right|_{0}=0$ and $\left.n_{z}\right|_{d}=0$. Strictly speaking this is true only in the case of "strong" superconductors and "weak" antiferromagnets: $\left|g_{S}\right| \gg\left|g_{A}\right|$. However, analysis of the general case shows that relaxing this condition does not change the qualitative picture.

At this point one can easily specify the analogy between our problem and the problem of a liquid crystal in a slab with anchoring walls, in an electric field. If the electric field is perpendicular to the walls, it will try to align the director of the liquid crystal along the field. At small voltages the field is unable to overcome the effect of surface pinning, and the equilibrium configuration remains uniform. However, with increasing voltage the system will undergo a Freedericksz transition, in which the director begins to align along the field. More interestingly, this transition is known to depend on the applied boundary conditions, i.e., on the relative twist of the anchoring directions on the two sides of the slab (the twisted nematic transition) [8].

We now show that similar effects arise in $S-A-S$ sandwiches within $\mathrm{SO}(5)$ theory. The role of the voltage is played by $d \sqrt{g_{A} / \rho}$, and the superconducting phase difference across the junction corresponds to the twist angle imposed by the two anchoring walls. The $S-A-S$ sandwiches will undergo a phase transition in which the $A$ region, between the two superconductors, goes from being purely superconducting (by virtue of the proximity effect) into a mixed $S / A$ state. We also show that, sufficiently close to such a Freedericksz transition, the system possesses nontrivial current-phase characteristics, as a consequence of the transition.

In the $A$ region the Euler-Lagrange equations for the functional (2) are

$$
\rho \frac{d^{2} \theta}{d x^{2}}+\rho \cos \theta \sin \theta\left(\frac{d \phi}{d x}\right)^{2}+2 g_{A} \sin \theta \cos \theta=0,
$$

$$
\frac{d}{d x}\left(\cos ^{2} \theta \frac{d \phi}{d x}\right)=0 .
$$

The boundary conditions for these equations are given by

$$
\begin{gathered}
\theta(x=0)=0, \quad \phi(x=0)=0, \\
\theta(x=d)=0, \quad \phi(x=d)=\Delta \Phi,
\end{gathered}
$$

where $\Delta \Phi$ is the phase difference between two superconductors.

Equation (4) is nothing but the conservation of current.

$$
I_{s}=n_{1} \partial_{x} n_{2}-n_{2} \partial_{x} n_{1}=\cos ^{2} \theta \frac{d \phi}{d x} .
$$

So we can write (3) as

$$
\rho \frac{d^{2} \theta}{d x^{2}}+\rho \sin \theta \frac{I_{s}^{2}}{\cos ^{3} \theta}+2 g_{A} \sin \theta \cos \theta=0 .
$$

The last equation can be easily integrated once giving

$$
\xi_{A}^{2}\left(\frac{d \theta}{d x}\right)^{2}=-\frac{I_{s}^{2} \xi_{A}^{2}}{\cos ^{2} \theta}-\sin ^{2} \theta+\frac{I_{s}^{2} \xi_{A}^{2}}{\cos ^{2} \theta_{0}}+\sin ^{2} \theta_{0}
$$

with the characteristic length

$$
\xi_{A}=\sqrt{\rho / 2 g_{A}} .
$$

In writing (8) we expressed the constant of integration in terms of the maximal value $\theta_{0}$ that will be reached at $x=d / 2$ (where $d \theta / d x=0$ ). This immediately results in an equation for $\theta_{0}$

$$
\begin{aligned}
\frac{d}{2 \xi_{A}} & =\int_{0}^{\theta_{0}} \frac{d \theta}{\sqrt{-\frac{\omega_{s}^{2}}{\cos ^{2} \theta}-\sin ^{2} \theta+\frac{\omega_{s}^{2}}{\cos ^{2} \theta_{0}}+\sin ^{2} \theta_{0}}} \\
& =\frac{\cos \theta_{0}}{\sqrt{\omega_{s}^{2}+\cos ^{2} \theta_{0}}} K(k),
\end{aligned}
$$

where $\omega_{s}=I_{s} \xi_{A}$, the parameter $k$ is defined by

$$
k^{2}=\frac{\sin ^{2} \theta_{0} \cos ^{2} \theta_{0}}{\omega_{s}^{2}+\cos ^{2} \theta_{0}}
$$

and $K$ is the complete elliptic integral of the first kind. Equation (10) should be supplemented by an equation for the current $\omega_{s}$ in terms of the phase difference across the junction 


$$
\begin{aligned}
\Delta \Phi & =2 I_{s} \int_{0}^{d / 2} \frac{d x}{\cos ^{2} \theta(x)}=2 I_{s} \int_{0}^{\theta_{0}}\left(\frac{d \theta}{d x}\right)^{-1} \frac{d \theta}{\cos ^{2} \theta} \\
& =2 \omega_{s} \int_{0}^{\theta_{0}} \frac{d \theta}{\cos ^{2} \theta \sqrt{-\frac{\omega_{s}^{2}}{\cos ^{2} \theta}-\sin ^{2} \theta+\frac{\omega_{s}^{2}}{\cos ^{2} \theta_{0}}+\sin ^{2} \theta_{0}}} \\
& =2 \omega_{s} \frac{\cos \theta_{0}}{\sqrt{\omega_{s}^{2}+\cos ^{2} \theta_{0}}} \Pi_{1}\left(-\sin ^{2} \theta, k\right) .
\end{aligned}
$$

Here $\Pi_{1}(n, k)$ is a complete elliptic integral of the third kind.

One can easily see that Eq. (10) has a solution only when $d / \xi_{A} \geq \pi / \sqrt{1+\omega_{s}^{2}}$. For smaller $d$ the only solution will be $\theta_{0}=0$, which means that the $A$ region remains uniformly superconducting. Even though antiferromagnetism would be favored in a bulk material of this kind, proximity to a "strong" superconductor forces it to be uniformly superconducting. When $d_{c}=\pi \xi_{A} / \sqrt{1+\omega_{s}^{2}}$, a second-order transition occurs at which for $d>d_{c} \theta_{0}$ starts to increase as $\sqrt{d-d_{c}}$, so that the $A$ region exhibits both kinds of order: superconductivity and antiferromagnetism. It is interesting to note that a nonzero $\omega_{s}$ decreases the critical width of the $A$ region. This can be understood as the result of having an extra "torque" in the $x-y$ plane. This result raises the very interesting possibility of choosing a width of the $A$ region below the critical value at zero current $d_{c 0}=\pi \xi$ and then tuning the system through the transition by simply passing a current through the junction.

In Fig. 3 we present such an example, for the case $d=$ $0.85 d_{c 0}$. This figure shows that the system undergoes a transition when $\Delta \Phi=1.7$. Below the transition, $\theta_{0}$ is identically zero and $I_{s}$ is a linear function of $\Delta \Phi$, as one would expect for a uniform superconductor. However, above the Freedericksz transition, $\theta_{0}$ starts to grow and $I_{s}$ vs $\Delta \Phi$ develops curvature. Eventually, at $\Delta \Phi=\pi$, $\theta_{0}=\pi / 2$ and $I_{s}$ goes to zero. We note that further interesting differences with the conventional proximity

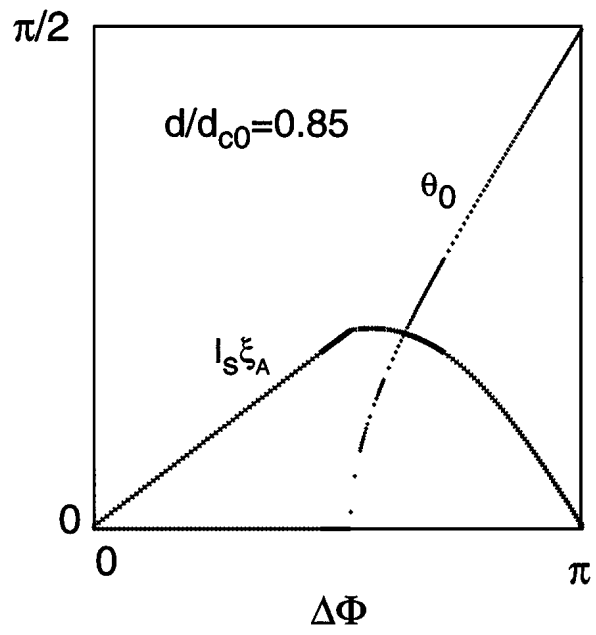

FIG. 3. $\theta_{0}$ vs $\Delta \phi$ and $I_{s}$ vs $\Delta \phi$ for an $S-A-S$ junction. The onset of $\theta_{0}$ and the discontinuity in slope of $I_{s}$ both occur at the Freedericksz transition. effect can be expected in the dynamical state at finite voltages. In the presence of a finite voltage across the junction, the full $\mathrm{SO}(5)$ order parameter will undergo periodic motion in $\mathrm{SO}(5)$ space, permitting exploration of the low $q$-vector dynamics of $\mathrm{SO}(5)$ theory.

Figure 4 shows that the feature, $\theta_{0}=\pi / 2$ when $\Delta \Phi=\pi$, occurs for all widths of the $A$ region. It may be understood as follows: The energy required to twist the superconducting order parameter by $\pi$ without changing its magnitude is the same as the energy required to rotate the superspin into the antiferromagnetic plane and back into the superconducting plane. However, rotating the superspin into the antiferromagnetic direction allows the system to lower its energy because of the $g$ term.

This effect is an interesting $\mathrm{SO}(5)$ analog of the result of Krotov et al. [9] that superconductivity between antiferromagnetic stripes is suppressed for nontopological stripes and enhanced for topological stripes.

Figure 5 illustrates the nontrivial current-phase characteristics of $S-A-S$ junctions with increasing width of the $A$ layer. When $d<d_{c 0}$ they show a transition from linear dependence below the transition to sin-like dependence above it. Some asymmetry persists in the curves for $d \geq$ $d_{c o}$, and for $d \gg d_{c 0}$ they show the usual $\sin (\Delta \Phi)$ dependences of superconducting-insulating-superconducting $(S-I-S)$ junctions.

It is easy to calculate the critical current of our junctions. For a given $d$, Eq. (10) does not have any solution for currents that are too large. The first solution appears at a point that corresponds to the maximum of $k^{2}$ in Eq. (11)

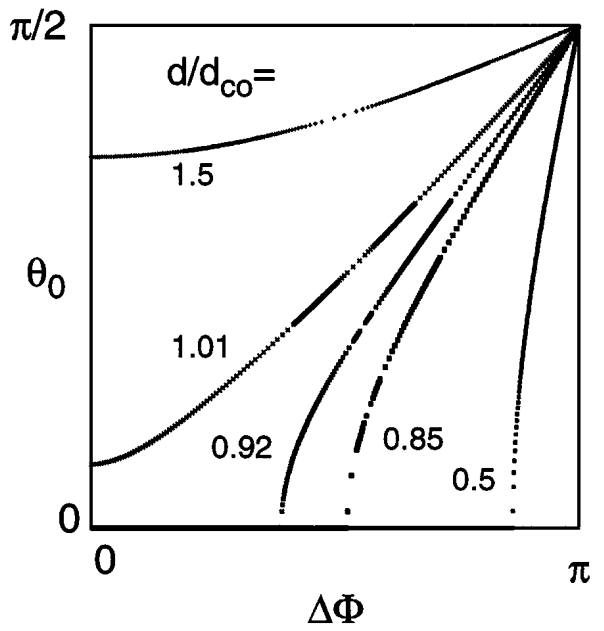

FIG. 4. $\theta_{0}$ vs $\Delta \Phi$ for junctions with different $d / d_{c 0}$. Notice that for $\Delta \Phi=\pi$ we always have $\theta_{0}=\pi / 2$. 


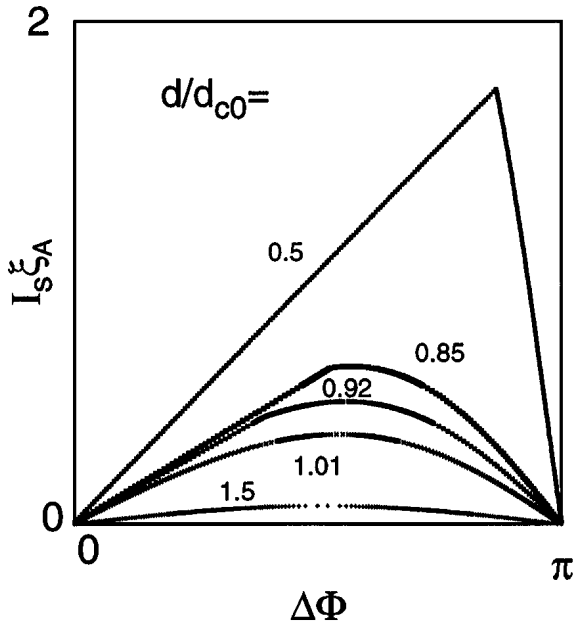

FIG. 5. Current-phase characteristics of junctions with different $d / d_{c 0}$.

[10]. This $k_{\max }$ is given by

$$
k_{\max }^{2}=1-2 \omega_{s}\left(\sqrt{1+\omega_{s}^{2}}-\omega_{s}\right) \simeq 1-2 \omega_{s} .
$$

Using the asymptotic forms of the elliptic functions, we find for Eq. (10) $d /\left(2 \xi_{A}\right)=\ln \left(4 / \sqrt{2 \omega_{s}}\right)$ which gives the critical current

$$
I_{s} \approx \frac{8}{\xi_{A}} e^{-d / \xi_{A}} .
$$

So $\xi_{A}$ represents a new correlation length for superconducting proximity effects across antiferromagnets: According to [1] $g_{A}=2 \chi\left(\mu_{c}^{2}-\mu^{2}\right)$. Then from Eq. (9) we see that, when $\mu$ is close to $\mu_{c}$ (and hence $g_{A}$ is small), $\xi_{A}$ will be large. This could provide a new and natural explanation of the long range proximity effect sometimes observed in $\mathrm{PrBa}_{2} \mathrm{Cu}_{3} \mathrm{O}_{7}$ (PBCO) [3-5]. We note, however, that asymmetry in the $\chi$ 's will generate a cutoff for $\xi_{A}$ in Eq. (9). For $\chi_{c}>\chi_{\pi}$ we find $\xi_{\max }=\sqrt{\rho / \eta}$ where $\eta=2 \mu_{c}^{2}\left(\chi_{c}-\chi_{\pi}\right)$.

Other effects that we have not considered here may provide an extra cutoff for Josephson coupling in PBCO materials on length scales shorter than given by Eq. (9). An example may be thermal decoherence which puts an upper bound on the coherence length $\xi_{A} \leq \rho_{s} / T$. However, our calculations show that unlike the case of Josephson coupling across "conventional" insulators, where the correlation length is set by $h v_{F} / \Delta_{\text {ins }}$, with $\Delta_{\text {ins }}$ being the energy gap in the insulating material and $v_{F}$ the Fermi velocity in the superconducting material, in superconducting proximity effects across $\mathrm{SO}(5)$ antiferromagnets the superconducting order does not have to vanish on the atomic length scale [11].

The effects considered in this paper are not necessarily restricted to the $\mathrm{SO}(5)$ nonlinear sigma model. One may think of a general Ginzburg-Landau theory with antiferromagnetic and superconducting order parameters like those considered in [12]. The Freedericksz transition is, of course, the result of having two states close in energy, and it may be present even within mean field theories that con- sider general competition between spin-density wave and dSC states. The appearance of the long range proximity effect is more subtle. Even within the $\mathrm{SO}(5)$ nonlinear sigma model, one may have either a first-order transition or a coexistence region bounded by two second-order phase transitions. The subtlety of the isotropic sigma model (here what matters is not the isotropy of $\rho$, but equality of charge and $\pi$ compressibilities; see [1] for details) lies in the fact that it separates the two sectors. So, it is as much a firstorder line as two coinciding second-order lines. The diverging length scale is the result of this closeness to the second-order transition. Therefore, we think that having a second order phase transition close by is what is important for the long correlation length in the antiferromagnet. Mean-field theories that have such a transition may also lead to the long range proximity effect.

The authors are greatly indebted to S.C. Zhang for numerous illuminating discussions on the $\mathrm{SO}(5)$ theory and for generously sharing of his ideas. We would like to thank A. Fetter for drawing our attention to [8]. A.J.B. and C.K. acknowledge support by the Natural Sciences and Engineering Research Council of Canada and by the Ontario Centre for Materials Research. C.K. also acknowledges support from the Guggenheim Foundation. This work is also supported by the Office of Naval Research and the National Science Foundation through the NSF-MRSC program.

[1] S. C. Zhang, Science 275, 1089 (1997).

[2] P.M. Chaikin and T.C. Lubensky, Principles of Condensed Matter Physics (Cambridge University Press, Cambridge, England, 1995).

[3] Y. Suzuki et al., Phys. Rev. Lett. 73, 328 (1993).

[4] J. B. Barneri et al., Appl. Phys. Lett. 59, 742 (1991).

[5] T. Hashimoto et al., Appl. Phys. Lett. 60, 1756 (1992).

[6] D. Arovas, J. Berlinsky, C. Kallin, and S.C. Zhang, Phys. Rev. Lett. 79, 2871 (1997).

[7] The $\mathrm{SO}(5)$ theory assumes that $T_{c}$ of the underdoped cuprates is determined by orientational (phase) fluctuations and not by mean-field transition temperature. This justifies the use of our London-type equation (1) even at finite temperature.

[8] Another analog of the system under consideration is orientational transition in ${ }^{3} \mathrm{He}-A$ in a slab. See, for example, D. Vollhardt and P. Wolfle, The Superfluid Phases of Helium 3 (Taylor \& Francis, London, 1990).

[9] Yu. A. Krotov, D.H. Lee, and A. V. Balatsky, Report No. cond-mat/9705031.

[10] This simple argument applies only when $\omega_{s}$ is much smaller than one, i.e., well above the Freedericksz transition. For smaller widths one can expect small corrections due to the prefactor of the elliptic function in (10).

[11] Another important effect which is not sufficiently illuminated in the current paper and which may in principle lead to shorter cutoff on the correlation length than our Eq. (9) is the effect of fermions.

[12] G. Psaltakis and E. Fenton, Solid State Phys. 16, 3913 (1983); K. Machida and M. Kato, Phys. Rev. Lett. 58, 1986 (1987). 